\title{
Clinical study of acupuncture treatment on motor aphasia after stroke
}

\author{
Qian $\mathrm{Wu}^{\mathrm{a}, \mathrm{d}, 1}$, Xiaoqing $\mathrm{Hu}^{\mathrm{a}, 1}$, Xiuyun $\mathrm{Wen}^{\mathrm{b}}$, Fuming $\mathrm{Li}^{\mathrm{c}, *}$ and Wenbin $\mathrm{Fu}^{\mathrm{d}, *}$ \\ ${ }^{a}$ The Second Clinical Medical College, Guangzhou University of Chinese Medicine, Guangzhou, \\ Guangdong, China \\ ${ }^{\mathrm{b}}$ Baoan Hospital, Southern Medical University, Shenzhen, Guangdong, China \\ ${ }^{\mathrm{c}}$ Jinshazhou Hospital of Guangzhou University of Chinese Medicine, Guangzhou, Guangdong, China \\ ${ }^{\mathrm{d}}$ Department of Acupuncture and Moxibustion, Guangdong Provincial Hospital of TCM, Guangzhou, \\ Guangdong, China
}

\begin{abstract}
.
OBJECTIVE: To study the safety and effectiveness of the Heart-Gallbladder acupuncture treatment for motor aphasia after stroke via clinical studies.

METHODS: Sixty valid patients were divided into two groups randomly with a ratio of 1:1. The treatment group was the Heart-Gallbladder acupuncture group and the control group was the conventional acupuncture group. The two groups underwent testing before and after treatments, which included: the Aphasia Battery of Chinese (ABC), the Chinese functional communication profile (CFCP), and the Boston diagnostic aphasia examination (BDAE).

RESULTS: All the BDAE, $\mathrm{CFCP}$ and $\mathrm{ABC}$ results showed a significant difference between the two groups after treatment $(P<0.05)$, indicating that the Heart-Gallbladder acupuncture treatment for motor aphasia after stroke can reduce the degree of aphasia and improve patients' daily communication skills more than the conventional acupuncture treatment. The HeartGallbladder acupuncture treatment is better than the conventional acupuncture treatments for motor aphasia after stroke, with significantly improved scores for fluency, repetition, naming, and reading.

CONCLUSIONS: Both the Heart-Gallbladder acupuncture and the conventional acupuncture are effective in the treatment of motor aphasia after stroke. Nevertheless, when compared to the conventional acupuncture, the Heart-Gallbladder acupuncture had better efficacy and it is safe as well.
\end{abstract}

Keywords: Motor aphasia, stroke, Heart-Gallbladder treatment, tongue acupuncture

\section{Introduction}

Cerebrovascular disease is identified as a major detriment to human health by the World Health Organization (WHO). Stroke causes 9\% of all deaths worldwide [1,2]. Approximately 50\% to $70 \%$ of all patients with cerebrovascular disease suffered from paralysis [3], creating mental and economic burdens on society and family. Language disorders occur more frequently in various sequelae of cerebrovascular disease. Research shows $21 \%$ to $38 \%$ of stroke patients suffer from aphasia [4-7]. Most domestic scholars believe that about one-third of stroke patients deal with continuous sequelae, such as aphasia [8].

\footnotetext{
${ }^{1}$ These authors contributed equally.

${ }^{*}$ Corresponding authors: Wenbin Fu and Fuming Li, Department of Acupuncture and Moxibustion, Guangdong Provincial Hospital of TCM, Guangzhou, Guangdong, China. Tel.: +86 13808888626; E-mail: fuwenbin@139.com.
}

0928-7329/16/\$35.00 @ 2016 - IOS Press and the authors. All rights reserved This article is published online with Open Access and distributed under the terms of the Creative Commons Attribution NonCommercial License. 
Motor aphasia is a type of aphasis caused by damaging lesions on the left posterior inferior frontal gyrus and adjacent regions. These patients maintain their ability to comprehend and ideate, but the application of the language is decreased significantly. In this study, we develop a clinical protocol to determine whether the acupuncture treatment based on Heart-Gall bladder regulating was effective and safe for the treatment of motor aphasia after stroke.

\section{Methods}

\subsection{Participants}

The study was conducted at the Department of Acupuncture and Moxibustion, Guangdong Provincial Hospital of Traditional Chinese Medicine (TCM). Sixty participants were diagnosed with motor aphasia by the Department of Acupuncture and Moxibustion and the Department of Encephalopathy. Participants were recruited from January 2012 to October 2013. Characteristics such as gender, age, education level, staging of course of disease, lesion nature, (cerebral hemorrhage or cerebral infarction), and stroke frequency, were analyzed using Aphasia Battery of Chinese (ABC), Chinese functional communication profile (CFCP), and Boston diagnostic aphasia examination (BDAE) before and after treatment.

\subsection{Diagnostic criteria}

Diagnostic criterion for stroke was in accordance with The Fourth National Conference of Cerebral Vascular Disease by China Medical Association (CMA) in 1995. The TCM diagnostic criteria standard for the diagnosis and therapeutic effects of stroke in traditional Chinese medicine was by SATCM in 1996. The diagnostic criteria for motor aphasia based on the Aphasia Battery of Chinese (ABC) was also used.

\subsection{Case inclusion and exclusion criteria}

\subsubsection{Inclusion criteria}

1. Ages 35-80 years old, stable diseases, no severe complications observed 2. Corresponding to the diagnosis standards of stroke above 3. Having a normal IQ, language function and hearing before the onset, being conscious, and willing to cooperate after the onset 4 . Informed consent.

\subsubsection{Exclusion criteria}

1. Not corresponding to the diagnosis standards and inclusion standards 2. Severe hearing disorders and visual disturbance 3. Patients with cerebrovascular disease after imaging examination 4. Combined with severe primary diseases of liver, kidney, hematopoietic system and endocrine system 5. Exclusion of aphasia caused by lesions of phonation organs

\subsection{Design of treatment group}

\subsubsection{Location of acupoints}

Patients assigned to the treatment group were needled at the following points: NeiGuan (PC-6, bilateral), YangLingquan (GB-34, bilateral), heart (tongue acupuncture), gallbladder (tongue acupuncture). NeiGuan (PC-6, bilateral) and YangLingquan (GB-34, bilateral) were determined using the "Meridian and Acupuncture" textbook of the eleventh five-year plan. Tongue acupuncture was determined by the 2002 "Collection of Master Guan's Acupuncture Experience". 


\subsubsection{Operation method}

Patients were maintained in the supine position. NeiGuan (PC-6, bilateral), YangLingQuan (GB-34, bilateral) were needled using sterilized disposable needles $(0.30 \mathrm{~mm}$ in diameter and $25 \mathrm{~mm}$ in length) from the Huatuo brand. Heart (tongue acupuncture) and gallbladder (tongue acupuncture) were needled using sterilized disposable needles $(0.30 \mathrm{~mm}$ in diameter and $50 \mathrm{~mm}$ in length) from the Huatuo brand. Routine operation after disinfection was a follows: NeiGuan (PC-6) were needled before Yanglingquan (GB-34); Neiguan (PC-6) and Yanglingquan (GB-34) were inserted at a depth of 10-20 mm according to the location of point and the somatotype of patients. Practitioners twisted the needles with the puncturing method of rapid lifting thrusting $(120 \mathrm{r} / \mathrm{min})$ and twirling in small amplitude to achieve "Deqi" sensation (described as numb, distended and aching). The needles were left in for 25 mins and then removed. Approximately $3 \%$ Hydrogen peroxide or potassium permanganate was given to participants to gargle with in order to clean the mouth before tongue acupuncture was performed. Acupoints were inserted at a depth of 1-2 mm. Practitioners twisted and thrusted the needles with rapid lifting thrusting $(100 \mathrm{r} / \mathrm{min})$ for one min, then removed the needles. Practitioners would observe whether hemorrhaging of the tongue occurred after the needles were removed. If patients appeared to exhibit bleeding at the tongue acupoint after the needling, applying pressure with a sterile cotton ball.

\subsubsection{Course of treatment}

Patients from the two groups received treatment once a day, 5 times a week, totaling 3 weeks.

\subsection{Control group}

\subsubsection{Location of acupoints}

Patient assigned to the control group were needled at the following points: JinJin, YuYe, and TongLi (HT-5, bilateral). Acupoints were determined using the "Meridian and Acupuncture" textbook of the eleventh five-year plan. Selection of the control group was based on Eleven-Five programming teaching material of meridians acupoints. The operation method and course of treatment were the same as the treatment groups.

\subsection{Measures}

The following measures were used: 1. Aphasia Battery of Chinese (ABC). 2. The Chinese functional communication profile (CFCP) 3. Boston diagnostic aphasia examination (BDAE).

\subsection{Statistical analysis}

We used SPSS version17.0 to perform all the statistical analyses. Intention-to treat was applied to deal with the data. Baseline differences between participants in the treatment group and control group were examined using chi-square test or Mann-Whitney Test. We assumed that $P<0.05$ was taken to indicate statistical significance.

\section{Results}

Five of the 60 participants withdrew during study period, two of whom were from the treatment group, and three from the standard group. The dropout rate between the two groups had no significant difference. The two groups of the demographic characteristics base line were balanced (Table 1). The results 
Table 1

Demographic characteristics

\begin{tabular}{|c|c|c|c|c|c|c|c|c|c|c|c|}
\hline \multirow[t]{2}{*}{ Group } & \multirow[t]{2}{*}{ Case } & \multicolumn{2}{|c|}{ Sex } & \multirow[t]{2}{*}{ Age } & \multicolumn{3}{|c|}{ Courses } & \multicolumn{2}{|c|}{ Stroke type } & \multicolumn{2}{|c|}{ Stroke times } \\
\hline & & M & $\mathrm{F}$ & & $\begin{array}{l}\text { Acute } \\
\text { stage }\end{array}$ & $\begin{array}{c}\text { Recovery } \\
\text { stage }\end{array}$ & $\begin{array}{l}\text { Chronic } \\
\text { stage }\end{array}$ & $\begin{array}{l}\text { Ischemic } \\
\text { stroke }\end{array}$ & $\begin{array}{l}\text { Hemorrhagic } \\
\text { stroke }\end{array}$ & $\begin{array}{l}\text { First } \\
\text { time }\end{array}$ & $\begin{array}{l}2 \text { times } \\
\text { or more }\end{array}$ \\
\hline Treatment group & 30 & 19 & 11 & $67.30 \pm 9.82$ & 6 & 14 & 10 & 21 & 9 & 22 & 8 \\
\hline Control group & 30 & 19 & 11 & $63.37 \pm 10.7$ & 11 & 10 & 9 & 21 & 9 & 20 & 10 \\
\hline
\end{tabular}

Table 2

$\mathrm{ABC}$ score between the 2 groups $(\bar{x} \pm s)$

\begin{tabular}{|c|c|c|c|c|c|c|}
\hline \multirow[t]{2}{*}{ Project } & \multicolumn{2}{|c|}{ Treatment group } & \multicolumn{2}{|c|}{ Control group } & \multirow[t]{2}{*}{$\mathrm{t}$} & \multirow[t]{2}{*}{$p$} \\
\hline & Before treatment & After treatment & Before treatment & After treatment & & \\
\hline Information & $6.93 \pm 2.56$ & $8.86 \pm 2.61$ & $6.80 \pm 2.61$ & $7.53 \pm 2.71$ & 1.94 & 0.06 \\
\hline Verbal fluency & $9.96 \pm 2.47$ & $15.83 \pm 4.1$ & $10.20 \pm 1.65$ & $12.76 \pm 2.11$ & 3.64 & 0 \\
\hline Understanding & $183.66 \pm 41.9$ & $197.40 \pm 41.6$ & $189.43 \pm 31.16$ & $197.76 \pm 35.45$ & 0.034 & 0.97 \\
\hline Retelling & $20.40 \pm 9.49$ & $44.60 \pm 16.71$ & $18.26 \pm 8.1$ & $29.00 \pm 14.91$ & 3.82 & 0 \\
\hline Naming & $14.86 \pm 5.71$ & $38.63 \pm 13.24$ & $15.66 \pm 4.83$ & $26.83 \pm 11.03$ & 3.75 & 0 \\
\hline Reading & $47.86 \pm 12.49$ & $69.10 \pm 17.95$ & $45.36 \pm 12.14$ & $56.10 \pm 15.06$ & 3.03 & 0 \\
\hline
\end{tabular}

Table 3

CFCP score between the 2 groups $(\bar{x} \pm s)$

\begin{tabular}{lccccc}
\hline Group & Total cases & Before treatment & After treatment & $\mathrm{t}$ & $\mathrm{p}$ \\
\hline Treatment group & 30 & $156.66 \pm 40.37$ & $200.00 \pm 42.59$ & 8.64 & 0 \\
Control group & 30 & $152.33 \pm 38.39$ & $171.66 \pm 46.09$ & 2.30 & 0.03 \\
\hline
\end{tabular}

Table 4

The segmented comparison of BDAE score between 2 groups $(\mathrm{G}=$ Grade $)$

\begin{tabular}{lccccrrr}
\hline Group & Cases & G0 & G1 & G2 & G3 & G4 & G5 \\
\hline Before treatment of the Treatment group & 30 & 0 & 2 & 7 & 13 & 8 & 0 \\
After treatment of the Treatment group & 30 & 0 & 1 & 2 & 8 & 11 & 8 \\
Before treatment of the control group & 30 & 0 & 3 & 9 & 11 & 7 & 0 \\
After treatment of the control group & 30 & 0 & 3 & 4 & 10 & 11 & 2 \\
\hline
\end{tabular}

were shown as followed in Tables 1-5. As to Table 2, No significant differences between the two groups are found before treatment $(P>0.05)$. The difference between the comparisons within two groups is statistically significant according to the T-tests $(P<0.05)$. The score of verbal fluency, retelling, naming and reading improved significantly in the treatment group. The difference between the two groups after treatment is statistically significant $(P<0.05)$. However, the score for information and understanding is no significant difference between the two groups $(P>0.05)$. As to Table 3 , before treatment, the CFCP score of the comparison between two groups is no significant difference $(P>0.05)$. After treatment, the difference between the comparison within the treatment group $(P<0.05)$, and within the control group $(P<0.05)$, is statistically significant. After treatments, the difference between the two groups, is also significant $(P<0.05)$. As to Table 4 , after treatment, the difference between the comparison within treatment group with Ridit test $(P<0.05)$, is statistically significant. After treatment, the difference between the comparison within control group with Ridit test $(P>0.05)$, is not statistically significant. After treatment, the difference between two groups with Ridit test $(P<0.05)$, is statistically significant. 
Table 5

The difference of clinical efficacies between the two groups

\begin{tabular}{lclcccc}
\hline Group & Total & Ineffective & Progress & Effective & Marked effect & Total effective rate \\
\hline Treatment group & 30 & $3(10 \%)$ & $4(13.33 \%)$ & $11(36.67 \%)$ & $12(40 \%)$ & $90 \%$ \\
Control group & 30 & $8(26.67 \%)$ & $13(43.33 \%)$ & $8(26.67 \%)$ & $1(3.33 \%)$ & $73.33 \%$ \\
\hline
\end{tabular}

\section{Safety and side effects}

In the study, the stroke patients were all given the basic therapy found in the China Guidelines on Prevention and Management of Cerebrovascular Disease. During the process of cerebral infarction and cerebral hemorrhage, the general condition of the patients can be controlled without acute acupuncture accidents. No acupoint infections, or needle syncope, occurred in either group.

\section{Discussion}

Guan's tongue acupuncture is a special acupuncture treatment developed by Guan Zhengzai based on the relationship between tongue and viscera meridians in "The Inner Canon of Huangdi", as well as decades of clinical experiences. Based on the theory of viscera meridian, according to the correlation between diseases and tongue acupoints, the heart and gall bladder function can be regulated via heart gall bladder acupoints in the tongue. From the circulation of meridian, NeiGuan is the collateral point in the Jueyin Pericardium Channel of Hand which is connecting to the Hand Shaoyang channel. The Hand Shaoyang channel meets Foot Shaoyang channel at the head, thus NeiGuan can regulate the heart and gall bladder channels. The so called YangLingQuan in "The Inner Canon of Huangdi" is the XiaHe acupoint in ball gladder channel, is it also the JingHui (Muscles Meet Point) in the Eight Influential acupoints. In the combined treatment for viscera, the first choice for regulating gall-bladder function is through XiaHe, thus we can regulate gall-bladder via YangLingQuan, and further regulating the spirit. The function of JingHui also demonstrates that YangLingQuan is related to the dexterity and agility of muscle. According to "Shuowen", the word "Jing" is composed of "power" and "muscle", which means the muscle that can produce power. "Lingshu: meridians chapter" says: "Lip and tongue, the fundament of muscle." Thus the YangLingQuan, JingHui can be used to treat the dysfunction of tongue.

It is reported that it is easier for the stroke patients with aphasis to have depression then the patients without aphasis, and the author implied that the depression could be one of the factors that influence the efficacy of acupuncture. Due to current limitation, we have not developed any experiment design with respect to depression, and we are hoping to deepen the related research in the future.

\section{Conclusion}

According to the $\mathrm{ABC}$ scores, there is a significant difference between the before and after treatment of the treatment group $(P<0.05)$, with a total effectiveness of $90.00 \%$. There is also a significant difference between before and after treatment of the control group $(P<0.05)$, with a total effectiveness of $73.33 \%$. There is also a significant difference between the control group and the treatment group after treatment $(P<0.05)$. The above results indicate that the Heart-Gallbladder acupuncture treatment is better than the conventional acupuncture treatment for motor aphasia after stroke, with significantly 
improved scores for fluency, repetition, naming, and reading. According to the CFCP scores, the treatment group before and after treatment received scores of $156.67 \pm 40.37$, and $200.00 \pm 42.59$, respectively. The control group before and after treatment received scores of $152.33 \pm 38.39$, and $171.67 \pm$ 46.09 , respectively. There is a significant difference between the two groups after treatment $(P<0.05)$. The results indicated that compared to the conventional acupuncture treatment, the Heart-Gallbladder acupuncture treatment for motor aphasia after stroke can improve patients' daily communication skills. According to the BDAE results for the treatment group, the number of persons before treatment were $0,2,7,13,8$, and 0 , receiving grades of $0-5$, respectively. The numbers after treatment were $0,1,2,8$, 11 , and 8 , respectively, and show a significant difference $(P<0.05)$. For the control group, the number of persons before treatment were $0,3,9,11,7$, and 0 , receiving grades of $0-5$, respectively. The numbers after treatment were $0,3,4,10,11$, and 2 , respectively, and show no significant difference $(P>$ $0.05)$. There is, however, a significant difference between the two groups after treatment $(P<0.05)$. The results indicate that compared to the conventional acupuncture treatment, the Heart-Gallbladder acupuncture treatment for motor aphasia after stroke can reduce the degree of aphasia. Also, there was no abnormality displayed by either group before or after treatment.

\section{Acknowledgements}

The research work was supported by The Special Fund for Traditional Chinese Medicine techniques of Guangdong Provincial Hospital of TCM (Situling Acupuncture Academic thoughts and inheritance Research) No. YK2013BIN04 \& No.2014KT1303.

\section{References}

[1] Murray CJ, Lopez AD. Mortality by cause for eight regions of the world: Global Burden of Disease Study, Lancet. 1997; 349: 1269-1276.

[2] Mathers CD, Boerma T, Ma Fat D. Global and regional causes of death, Br Med Bull. 2009; 92: 7-32.

[3] Berthier ML, Garcia-Casares N, Walsh SF, Nabrozidis A, Ruiz DMR, Green C, Davila G, Gutierrez A, and Pulvermuller F. Recovery from post-stroke aphasia: lessons from brain imaging and implications for rehabilitation and biological treatments, Discov Med. 2011; 12(1): 275-89.

[4] Berthier ML. Poststroke aphasia: epidemiology, pathophysiology and treatment, Drugs Aging. 2005; 22(1): $163-82$.

[5] Wade DT, Hewer RL, David RM, Enderby PM. Aphasia after stroke: natural history and associated deficits, J Neurol Neurosurg Psychiatry. 1986; 49: 11-16.

[6] Elman RJ, Ogar J, Elman SH. Aphasia: Awareness, advocacy, and activism, Aphasiology. 2000; 14(5-6): 455-459.

[7] Simmons-Mackie N, Code C, Armstrong E, Stiegler L, Elman RJ. What is aphasia? Results of an international survey, Aphasiology. 2002; 16(8): 837-848.

[8] Pedersen PM, Vinter K, and Olsen TS. Aphasia after stroke: type, severity and prognosis. The Copenhagen aphasia study, Cerebrovasc Dis. 2004; 17(1): 35-43. 